\title{
Adaptive Enhancing of Fingerprint Image with Image Characteristics Analysis*
}

\author{
Eun-Kyung Yun, Jin-Hyuk Hong and Sung-Bae Cho \\ Dept. of Computer Science, Yonsei University, \\ Biometrics Engineering Research Center \\ 134 Shinchon-dong, Sudaemoon-ku, Seoul 120-749, Korea \\ \{ekfree,hjinh\}@sclab.yonsei.ac.kr, sbcho@cs.yonsei.ac.kr
}

\begin{abstract}
The quality of the fingerprint images greatly affects the performance of the minutiae extraction. In order to improve the performance of the system, many researchers have been made efforts on the image enhancement algorithms. If the adaptive preprocessing according to the fingerprint image characteristics is applied in the image enhancement step, the system performance would be more robust. In this paper, we propose an adaptive preprocessing method, which extracts five features from the fingerprint images, analyzes image quality with Ward's clustering algorithm, and enhances the images according to their characteristics. Experimental results indicate that the proposed method improves both the quality index and block directional difference significantly in a reasonable time.
\end{abstract}

\section{Introduction}

Fingerprint identification is one of the most popular biometric technologies which is used in criminal investigations, commercial applications and so on. The performance of a fingerprint image matching algorithm depends heavily on the quality of the input fingerprint images [1]. Acquisition of good quality images is very important, but due to some environmental factors or user's body condition, a significant percentage of acquired images is of poor quality in practice [2]. From the poor quality images many spurious minutiae may be created and many genuine minutiae may be ignored. Therefore an image enhancement algorithm is necessary to increase the performance of the minutiae extraction algorithm.

Many researchers have been making efforts in the investigation of fingerprint image quality. Hong et al., Ratha et al., Shen et al., and many researchers worked on this area with sine wave, wavelet scalar quantization, and Gabor filter. However, most of the quality checks have been used as a criterion, which determines image rejection,

* This work was supported by the Korea Science and Engineering Foundation (KOSEF) through the Biometrics Engineering Research Center(BERC) at Yonsei University. 
or a performance measurement of image enhancement algorithm. In this case, only images are filtered uniformly without respect to the character of images. If the adaptive filtering is performed through appropriate analysis of image quality, images can be enhanced more effectively.

This paper proposes an adaptive preprocessing method to improve image quality appropriately. The preprocessing is performed after distinguishing the fingerprint image quality according to its characteristics. It is an adaptive filtering according to oily/dry/neutral images instead of uniform filtering. In the first stage, several features are extracted for image quality analysis and they go into the clustering module. Then, the adaptive preprocessing is applied to produce good quality images on two dataset: NIST DB 4 and private DB from Inha University.

\section{Fingerprint Image Quality}

In general, the fingerprint image quality relies on the clearness of separated ridges by valleys and the uniformity of the separation. Although the change in environmental conditions such as temperature and pressure might influence a fingerprint image in many ways, the humidity and condition of the skin dominate the overall quality of the fingerprint [2]. Dry skin tends to cause inconsistent contact of the finger ridges with the scanner's platen surface, causing broken ridges and many white pixels replacing ridge structure (see Fig. 1 (c)). To the contrary the valleys on the oily skin tend to fill up with moisture, causing them to appear black in the image similar to ridge structure (See Fig. 1 (a)). Fig. 1 shows the examples of the oily, neutral and dry images, respectively.

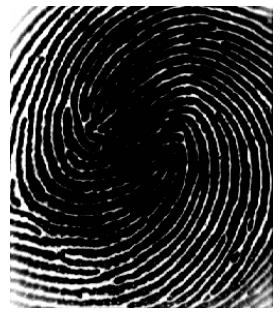

(a) Oily Image

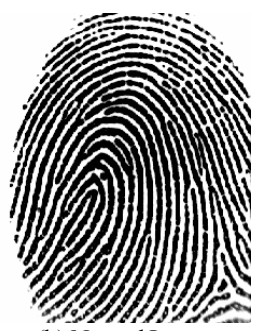

(b) Neutral Image

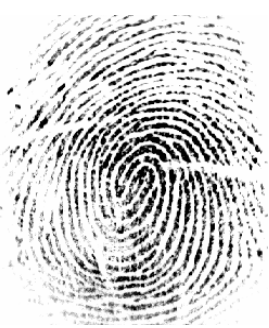

(c) Dry Image

Figure 1. Examples of fingerprint images.

- Oily image: Even though the separation of ridges and valleys is clear, some parts of valleys are filled up causing them to appear dark or adjacent ridges stand close to each other in many regions. Ridges tend to be very thick.

- Neutral image: In general, it has no special properties such as oily and dry. It does not have to be filtered.

- Dry image: The ridges are scratchy locally and there are many white pixels in the ridges. 
In this paper, the preprocessing is applied differently to the three types of image characteristics (oily/dry/neutral): For the oily images, valleys are enhanced by dilating thin and disconnected ones (valley enhancement process). For the dry images, ridges are enhanced by extracting their center lines and removing white pixels (ridge enhancement process) [3]. Most of the fingerprint identification systems preprocess images without considering their characteristics. If the preprocessing suitable for their characteristics is performed, much better images can be obtained.

\section{Adaptive Image Enhancement}

Fig. 2 shows the overview of the proposed system in this paper. For fingerprint image quality analysis, it extracts several features in fingerprint images using orientation fields, at first. Clustering algorithm groups fingerprint images with the features, and the images in each cluster are analyzed and preprocessed adaptively.

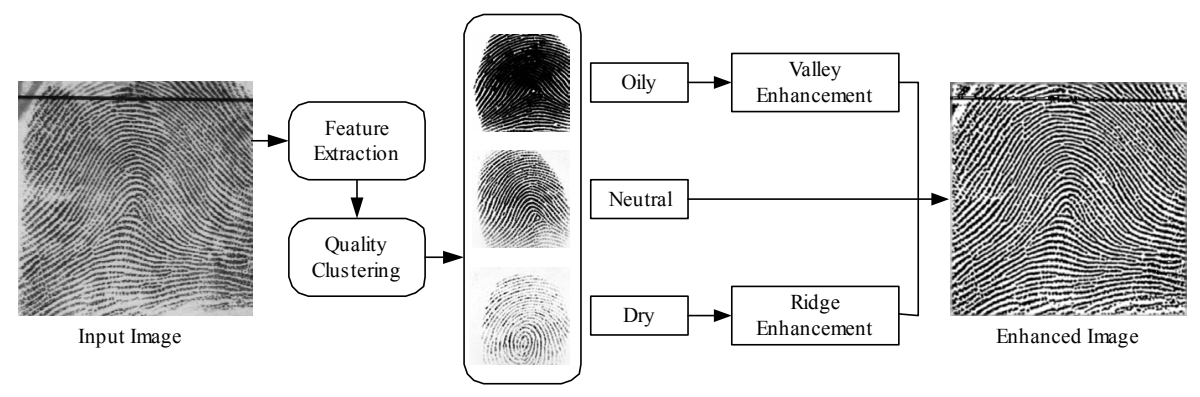

Figure 2. Overview of the proposed system

\subsection{Feature Extraction}

In this paper, five features are used to measure the image quality. The mean and variance of a gray-level fingerprint image are defined as follows.

$$
\begin{aligned}
& \text { Mean }=\frac{1}{N M} \sum_{i=0}^{N-1} \sum_{j=0}^{M-1} I(i, j) \\
& \text { Variance }=\frac{1}{N M} \sum_{i=0}^{N-1} \sum_{j=0}^{M-1}(I(i, j)-\text { Mean })^{2}
\end{aligned}
$$

The mean of gray values indicates the overall gray level of the image and the variance shows the uniformity of the gray values. $I(i, j)$ represents the intensity of the pixel at the $i$ th row and $j$ th column and the image $I$ is defined as an $N \times M$ matrix.

Fingerprint image can be divided into a number of non-overlapping blocks and block directional difference is computed [10]. Using the mask in Fig. 3, slit sum $S_{i}, i=$ $1, \ldots, 8$ is produced for center pixel $C$ of the block. 


\begin{tabular}{|l|l|l|l|l|l|l|l|l|}
\hline $\mathrm{P}_{51}$ & & $\mathrm{P}_{41}$ & & $\mathrm{P}_{31}$ & & $\mathrm{P}_{21}$ & & $\mathrm{P}_{11}$ \\
\hline & & & & & & & & \\
\hline $\mathrm{P}_{61}$ & & $\mathrm{P}_{52}$ & $\mathrm{P}_{42}$ & $\mathrm{P}_{32}$ & $\mathrm{P}_{22}$ & $\mathrm{P}_{12}$ & & $\mathrm{P}_{84}$ \\
\hline & & $\mathrm{P}_{62}$ & & & & $\mathrm{P}_{83}$ & & \\
\hline $\mathrm{P}_{71}$ & & $\mathrm{P}_{72}$ & & $\mathrm{C}$ & & $\mathrm{P}_{73}$ & & $\mathrm{P}_{74}$ \\
\hline & & $\mathrm{P}_{82}$ & & & & $\mathrm{P}_{63}$ & & \\
\hline $\mathrm{P}_{81}$ & & $\mathrm{P}_{13}$ & $\mathrm{P}_{23}$ & $\mathrm{P}_{33}$ & $\mathrm{P}_{43}$ & $\mathrm{P}_{53}$ & & $\mathrm{P}_{64}$ \\
\hline & & & & & & & & \\
\hline $\mathrm{P}_{14}$ & & $\mathrm{P}_{24}$ & & $\mathrm{P}_{34}$ & & $\mathrm{P}_{44}$ & & $\mathrm{P}_{54}$ \\
\hline
\end{tabular}

Figure 3. $9 \times 9$ Mask [10]

$$
\begin{gathered}
S_{i}=\sum_{i=1}^{8} P_{i j} \\
\text { Block directional difference }=\operatorname{Sum}\left(\left|S_{\max }-S_{\min }\right|\right)
\end{gathered}
$$

where $S_{\max }=\operatorname{Max}\left\{S_{i}, i=1, \ldots, 8\right\}$ and $S_{\min }=\operatorname{Min}\left\{S_{i}, i=1, \ldots, 8\right\}$.

$P_{i j}$ denotes the gray-level value of the $j$ th pixel in the direction $i . S_{\max }$ and $S_{\min }$ appear in each valley (white) pixel and in each ridge (black) pixel, respectively. Therefore, the directional difference of image block has a large value for good quality image blocks. In other words, ridge structures are characterized as well-separated. For bad quality image blocks, the directional difference of image block has a small value. Namely, ridge and valley are not distinguished in each other.

The ratio for ridge thickness to valley thickness is computed in each block [4]. Ridge thickness and valley thickness are obtained using gray level values for one image block in the direction normal to ridge flow. After that, the ratio of each block is computed and average value of the ratio is obtained over the whole image.

Orientation change is obtained by accumulating block orientation along each horizontal row and each vertical column of the image block. Orientation computation is as follows [5].

1) Divide $I$ into blocks of size $w \times w$.

2) Compute the gradients $\partial_{x}(i, j)$ and $\partial_{y}(i, j)$ at each pixel $(i, j)$ with the Sobel operator.

3) Estimate the local orientation of each block centered at pixel $(i, j)$ using the following equations [6]: 


$$
\begin{aligned}
& V_{x}(i, j)=\sum_{u=i-\frac{w}{2}}^{i+\frac{w}{2}} \sum_{v=j-\frac{w}{2}}^{j+\frac{w}{2}} 2 \partial_{x}(u, v) \partial_{y}(u, v) \\
& V_{y}(i, j)=\sum_{u=i-\frac{w}{2}}^{i+\frac{w}{2}} \sum_{v=j-\frac{w}{2}}^{j+\frac{w}{2}}\left(\partial_{x}^{2}(u, v) \partial_{y}^{2}(u, v)\right) \\
& \theta(i, j)=\frac{1}{2} \tan ^{-1}\left(\frac{V_{y}(i, j)}{V_{x}(i, j)}\right)
\end{aligned}
$$

where $\theta(i, j)$ is the least square estimate of the local ridge orientation at the block centered at pixel $(i, j)$. It represents the direction that is orthogonal to the direction of the Fourier spectrum of the $w \times w$ window. In this paper, we set $w=16$ and feature values are normalized between 0 and 1 .

\subsection{Image Quality Clustering}

As mentioned before, fingerprint image quality is divided into 3 classes, dry/neutral /oily. In this paper, we cluster images according to their characteristics using 5 features defined before. Fingerprint images are clustered by Ward's clustering algorithm which is one of the hierarchical clustering methods [7].

In this paper, image quality clustering tests on NIST DB 4 using five features described before. A total 2000 (a half of NIST DB) 5-dimensional patterns are used as input vectors of clustering algorithm. To determine the proper number of clusters, Mojena's cut-off value is used [8].

$$
\text { Mojena's Value }=h+\alpha s_{h}
$$

where $h$ is the average of dendrogram heights for all $N-1$ clusters and $s_{h}$ is the unbiased standard deviation of the heights. $\alpha$ is a specified constant and according to Milligan and Cooper [9], the best overall performance of Mojena's rule occurs when the values of $\alpha$ is 1.25 . For that reason, we set $\alpha=1.25$ as the number of clusters.

\subsection{Adaptive Preprocessing}

Smoothing is one of the conventional filtering methods [10]. It can remove the white pixels of ridges in case of dry images; however, it also removes necessary ridges that are thinner than neighbor ridges. Similarly, in case of oily images, it removes necessary valleys that are very thin while it removes black noises of valleys. Therefore, adaptive filtering with classifying image characteristics is better than uniform filtering. Fig. 4 shows a preprocessing method appropriate to image quality characteristics [3]. That is, ridges are enhanced in dry images and valleys are enhanced in oily images. 


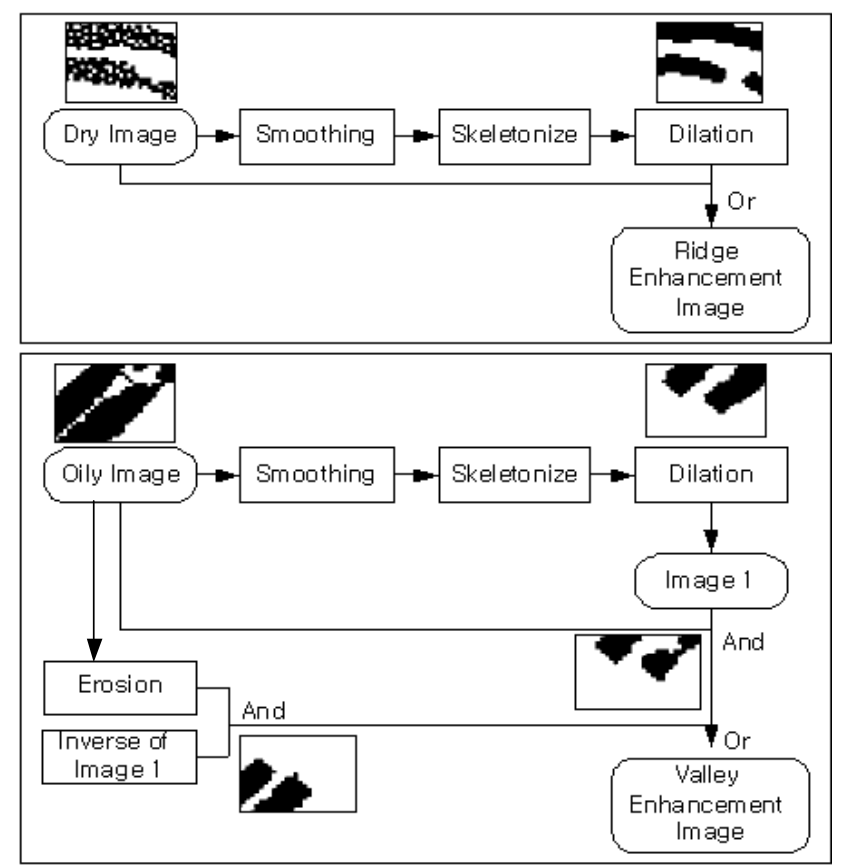

Figure 4. Preprocessing appropriate to image characteristics

1) Ridge enhancement of dry images: This extracts center lines of ridges and removes white pixels in ridges using this center-lined image. It also maintains the structure of the fingerprint.

A. Smoothing: smoothing is applied to the original image to reduce noises.

B. Thinning: a thinned image is obtained for extraction of ridge structures.

C. Dilation: a thinned image is dilated.

D. Extracting the union of black pixels in an original image and the image in $\mathrm{C}$ : white pixels in the ridges are removed. In this way, the ridge-enhanced image is obtained.

2) Valley enhancement of oily images: It is more complicated than ridge enhancement. It needs to detect regions where valleys are thin and disconnected. For this, thinning function extracts only the ridges thinner than a threshold. It means that the ridges wider than a threshold are eliminated.

A. Smoothing: it eliminates thin and disconnected valleys.

B. Thinning: thinned image using the threshold is obtained for extraction of ridge structures.

C. Dilation: dilated image is obtained and it contains the regions where ridges are sufficiently separated as black and the regions where ridges touch one another as white.

D. Composition of black pixels in the original image and in the image obtained in $\mathrm{C}$ : it detects the ridges whose thickness is wider than a threshold.

E. Composition of black pixels in the erosion of an original image and an 
inverse image of an image in $\mathrm{C}$

F. Extracting the union of black pixels of the images in D and E: in this way, the valley-enhanced image is obtained.

\section{Experiments}

The proposed method is verified with the NIST DB 4 (DB1) [11] and the highly controlled fingerprint DB at Inha University (DB2) [12]. DB1 consists of 4,000 fingerprint images (image size is $512 \times 480$ ) from 2,000 fingers. Each finger has two impressions. In DB2, the size of images is $248 \times 292$. Both of DB1 and DB2 are graylevel images. DB2 is used to check whether minutiae are extracted correctly or not. We use the first 2,000 fingerprint images in DB1 for clustering and the remaining 2,000 images for adaptive filtering using the rules obtained from the clustering results. Fingerprint image characteristics are analyzed using the Ward's clustering results. 30 clusters in a high rank appear in the dendrogram and according to Mojena's rule the proper number of clusters is 5 . Cluster 4 is assigned as dry, cluster 5 is oily and the remaining three clusters are neutral.

As a result, clustering made total 23 rules and Fig. 5 shows the essential rules. It indicates that in oily images ridges tend to be thicker than valleys and in dry images the ratio of ridge-valley thickness and mean are different from other clusters. In addition, the important factor of each feature is obtained by using the feature frequency in the rules. As shown in Table 1, the ridge-valley thickness ratio is the most important feature.

The image quality is measured in 2 different ways for quantitative analysis. First, block directional difference is used for quality check [11]. When the image quality is checked manually, we determine the image quality using the clearly separated ridges by valleys [4]. Hence, the block directional difference has a large value for good quality images. As shown in Fig. 6, the adaptive preprocessing is better than the uniform conventional filtering. The average values of the block directional difference with the adaptive enhancement are larger than those with the conventional filtering.

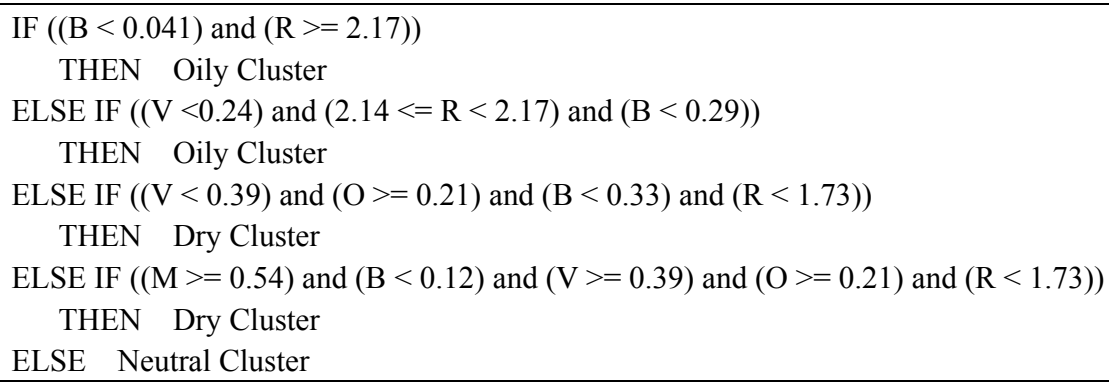

Figure 5. Rules obtained by clustering 
Table 1. Important factor of each feature

\begin{tabular}{cc}
\hline Feature & Important factor \\
\hline Mean $(\mathrm{M})$ & 0.67 \\
Variance $(\mathrm{V})$ & 0.20 \\
Block directional difference (B) & 0.37 \\
Orientation change (O) & 0.36 \\
Ridge-valley thickness ratio (R) & 1.00 \\
\hline
\end{tabular}

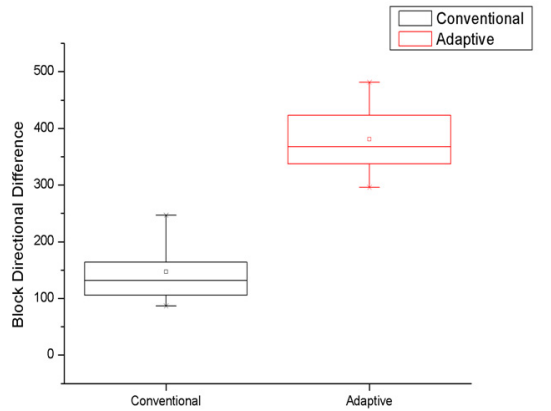

(a) Oily Images

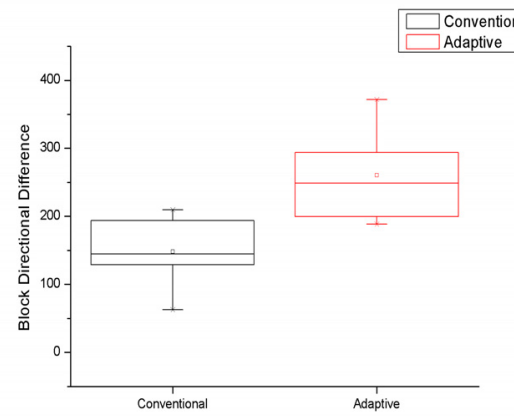

(b) Dry Images

Figure 6. Enhancement results with block directional difference

Second, the quality is measured with extracted minutiae. Image quality is assessed by comparing the minutiae set identified by human expert with that detected by minutiae extraction algorithm in an input fingerprint image. The larger the value of quality index, the better the minutiae extraction algorithm. Quality index is defined as follows:

$$
\text { quality index }=\frac{c}{c+f+u}
$$

where $c$ is the number of correctly detected minutiae, $f$ is the number of falsely detected minutiae, and $u$ is the number of undetected minutiae.

We use the 50 typical poor fingerprint images from DB2 to measure the filtering performance using extracted minutiae. First, we compute the Quality Index of the extracted minutiae with the conventional filtering and then the Quality Index of the extracted minutiae is computed with the adaptive filtering. Table 2 shows the Quality Index values of 50 typical images and the mean and variance of Quality Index values for all images. The Quality Index values with the adaptive enhancement are larger than those with the conventional filtering. Thus, it means that the adaptive preprocessing method improves the quality of the fingerprint images, which improves the accuracy of the extracted minutiae. To determine if there is a reliable difference between two means, we conduct a paired t-test. The calculated $t$-value (5.49) and $p$ value $(<0.0001)$ indicate that the difference between the two means is statistically very significant. That is, the quality difference between the conventional filtered images and adaptive filtered images is very significant in $99 \%$ confidence level. 
Table 2. The quality index values of fingerprint images: 50 typical images and the mean and variance

\begin{tabular}{ccc|ccc}
\hline Image \# & $\begin{array}{c}\text { Conventional } \\
\text { Filtering }\end{array}$ & $\begin{array}{c}\text { Adaptive } \\
\text { Filtering }\end{array}$ & Image \# & $\begin{array}{c}\text { Conventional } \\
\text { Filtering }\end{array}$ & $\begin{array}{c}\text { Adaptive } \\
\text { Filtering }\end{array}$ \\
\hline 1 & 0.16 & 0.37 & 27 & 0.11 & 0.18 \\
2 & 0.25 & 0.27 & 28 & 0.08 & 0.14 \\
3 & 0.0 & 0.25 & 29 & 0.03 & 0.06 \\
4 & 0.0 & 0.18 & 30 & 0.24 & 0.32 \\
5 & 0.07 & 0.1 & 31 & 0.07 & 0.13 \\
6 & 0.0 & 0.0 & 32 & 0.0 & 0.22 \\
7 & 0.0 & 0.24 & 33 & 0.34 & 0.32 \\
8 & 0.0 & 0.06 & 34 & 0.35 & 0.4 \\
9 & 0.12 & 0.14 & 35 & 0.06 & 0.22 \\
10 & 0.07 & 0.1 & 36 & 0.27 & 0.37 \\
11 & 0.17 & 0.2 & 37 & 0.38 & 0.42 \\
12 & 0.09 & 0.07 & 38 & 0.31 & 0.41 \\
13 & 0.15 & 0.22 & 39 & 0.33 & 0.22 \\
14 & 0.16 & 0.14 & 40 & 0.33 & 0.56 \\
15 & 0.23 & 0.4 & 41 & 0.27 & 0.41 \\
16 & 0.21 & 0.2 & 42 & 0.22 & 0.31 \\
17 & 0.22 & 0.16 & 43 & 0.22 & 0.45 \\
18 & 0.05 & 0.1 & 44 & 0.16 & 0.18 \\
19 & 0.12 & 0.19 & 45 & 0.11 & 0.18 \\
20 & 0.06 & 0.07 & 46 & 0.32 & 0.41 \\
21 & 0.22 & 0.1 & 47 & 0.02 & 0.11 \\
22 & 0.06 & 0.2 & 48 & 0.08 & 0.32 \\
23 & 0.02 & 0.05 & 49 & 0.11 & 0.12 \\
24 & 0.08 & 0.08 & 50 & 0.3 & 0.5 \\
\hline 25 & 0.28 & 0.25 & Mean & 0.1512 & 0.2226 \\
\hline 26 & 0.06 & 0.03 & Variance & 0.0130 & 0.0183 \\
\hline & & & & & \\
\hline
\end{tabular}

On the other hand, Fig. 7 shows some examples of enhanced images through the adaptive preprocessing. As shown in the figure, adaptively filtered images have better quality than conventionally filtered images.

Fig. 8 and 9 show some examples of enhanced images through the adaptive preprocessing. Fig. 8 shows the minutiae extracted in dry images with conventional filtering and adaptive filtering: (a) and (c) are with conventional filtering, (b) and (d) are with ridge enhancement filtering. While (a) and (c) have some falsely detected minutiae, endings, (b) and (d) have the correctly detected minutiae, bifurcations. Fig. 9 shows the minutiae extracted in oily images. While (a) and (c) with conventional filtering have falsely detected minutiae, bifurcations, or ridges connected, (b) and (d) with valley enhancement have correctly detected minutiae. 


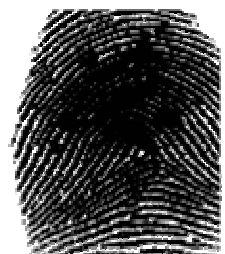

(a)

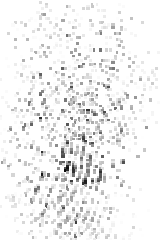

(d)

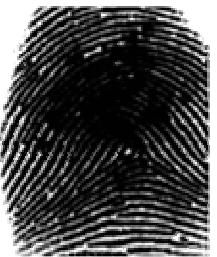

(b)

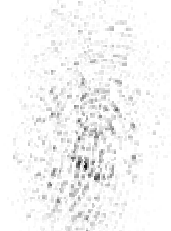

(e)

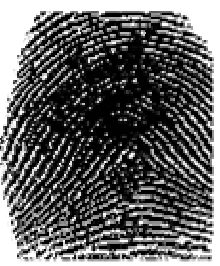

(c)

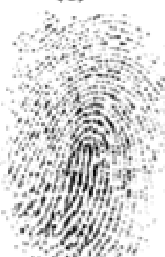

(f)

Figure 7. Examples of enhancement results. (a) and (d) are original oily and dry images, respectively. (b) and (e) are results of general filtering and (c) and (f) are results of adaptive filtering for (a) and (d), respectively.

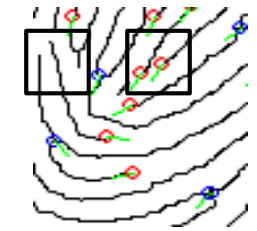

(a)

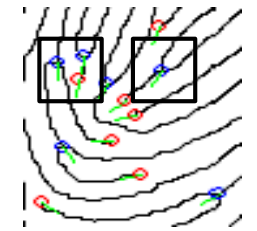

(b)

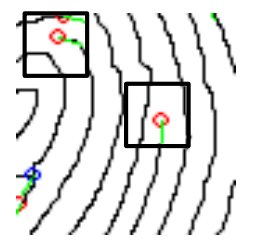

(c)

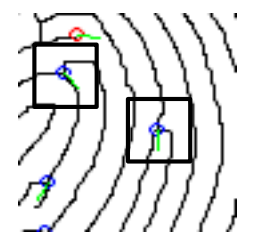

(d)

Figure 8. Dry image examples of minutiae extraction with conventional/adaptive filtering: (a) and (c) show the extracted minutiae with the conventional filtering, (b) and (d) show the extracted minutiae with ridge enhancement 


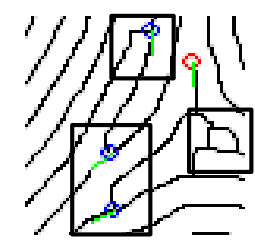

(a)

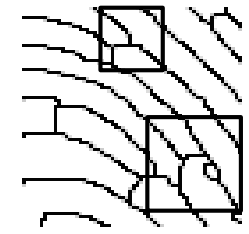

(c)

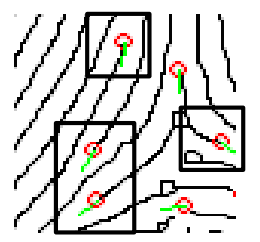

(b)

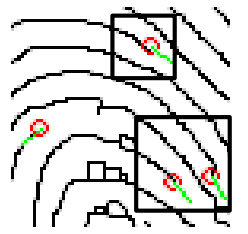

(d)

Figure 9. Oily image examples of minutiae extraction with conventional/adaptive filtering: (a) and (c) show the extracted minutiae with the conventional filtering, (b) and (d) show the extracted minutiae with valley enhancement

In order to incorporate the proposed preprocessing method into an online system, the whole process should be finished within a few seconds. Table 3 shows the time for each feature extraction and preprocessing.

Table 3. The time for the adaptive preprocessing (seconds) on Pentium 2GHz PC

\begin{tabular}{cccccc}
\hline $\mathrm{M} \& \mathrm{~V}$ & $\mathrm{~B}$ & $\mathrm{O}$ & $\mathrm{R}$ & Preprocessing & Total \\
\hline 0.001 & 0.141 & 0.063 & 0.047 & 0.301 & 0.553 \\
\hline
\end{tabular}

In order to incorporate the proposed preprocessing method into an online system, the whole process should be finished within a few seconds. Table 3 shows the time for each feature extraction and preprocessing.

\section{Concluding Remarks}

The performance of fingerprint identification system relies critically on the image quality. Hence, good quality image make the system performance more robust. However, it is very difficult to obtain good quality images in practical use. To overcome this problem, image enhancement step is required. But, most of the enhancement algorithms are applied equally to images without considering the image characteristics. Even though quality check is performed, it is not for quality analysis but for the performance evaluation of image enhancement algorithms or for checking whether an image is improved or not.

This paper has proposed an adaptive image enhancement method for fingerprint 
identification system. It is performed through the clustering of image quality characteristics. The performance of the proposed method was evaluated using the block directional difference and the Quality Index of the extracted minutiae. Experimental results show that the proposed method is able to improve both block directional difference and quality index, and the time required is in a reasonable range. Further works are going on to develop image characteristic factors for the identification system in real worlds.

\section{References}

1. N. K. Ratha, K. Karu, S. Chen, and A. K. Jain, "A Real-Time Matching System for Large Fingerprint Databases," IEEE Transaction on Pattern Analysis and Machine Intelligence, 18 (8), pp. 799-813, 1996.

2. L. C. Jain, U. Halici, I. Hayashi, S. B. Lee, and S. Tsutsui, Intelligent Biometric Techniques in Fingerprint and Face Recognition, CRC Press, 1999.

3. N. Ikeda, M. Nakanishi, K. Fujii, T. Hatano, S. Shigematsu, T. Adachi, Y. Okazaki and H. Kyuragi, "Fingerprint Image Enhancement by Pixel-Parallel Processing," 16th International Conference on Pattern Recognition, pp. 752-755, 2002.

4. E. Lim, X. Jiang and W. Yau, "Fingerprint Quality and Validity Analysis," IEEE International Conference on Image Processing, 1, pp. 22-25, 2002.

5. K. Karu, and A. Jain, "Fingerprint Classification," Pattern Recognition, 29 (3), pp. 389-404, 1996.

6. A. Rao, A Taxonomy for Texture Description and Identification, New York, NY: Springer-Verlag, 1990.

7. J. H. Ward, "Hierarchical Grouping to Optimize an Objective Function," Journal of the American Statistical Association, 58 (301), pp. 236-244, 1963.

8. R. Mojena, "Hierarchical Grouping Methods and Stopping Rules: An Evaluation," Computer Journal, 20, pp. 353-363, 1977.

9. G. W. Milligan and K. C. Cooper, "An Examination of Procedures for Determining the Number of Clusters in a Data Set," Psychometrika 50, pp. 159-179, 1985.

10. F. Galton, Finger Prints, Macmillan, London, 1892.

11. C. I. Watson and C. L. Wilson, NIST Special Database 4, Fingerprint Database. U. S. Nat'l Inst. of Standards and Technology, 1992.

12. H. Kang, B. Lee, H. Kim, D. Shin, and J. Kim, "A Study on Performance Evaluation of Fingerprint Sensors," The 4th International Conference Audio- and Video-based Biometric Person Authentication, pp. 574-583, 2003. 